OPEN ACCESS

Edited by:

Nam-Joon Cho,

Nanyang Technological University,

Singapore

Reviewed by:

Margarita Sánchez-Domínguez, Centro de Investigación en Materiales

Avanzados, Mexico

Dongxiang Li,

Qingdao University of Science and

Technology, China

*Correspondence:

Susanne K. Wiedmer

susanne.wiedmer@helsinki.fi

Specialty section:

This article was submitted to Colloidal Materials and Interfaces,

a section of the journal

Frontiers in Materials

Received: 30 May 2018 Accepted: 06 February 2019 Published: 01 March 2019

Citation:

Duša F, Chen W, Witos J and Wiedmer SK (2019) Calcium Dependent Reversible Aggregation of Escherichia coli Biomimicking Vesicles

Enables Formation of Supported Vesicle Layers on Silicon Dioxide.

Front. Mater. 6:23.

doi: 10.3389/fmats.2019.00023

\section{Calcium Dependent Reversible Aggregation of Escherichia coli Biomimicking Vesicles Enables Formation of Supported Vesicle Layers on Silicon Dioxide}

\author{
Filip Duša ${ }^{1}$, Wen Chen ${ }^{2}$, Joanna Witos ${ }^{3}$ and Susanne K. Wiedmer ${ }^{2 *}$ \\ ${ }^{1}$ Institute of Analytical Chemistry of the Czech Academy of Sciences, Brno, Czechia, ${ }^{2}$ Department of Chemistry, University of \\ Helsinki, Helsinki, Finland, ${ }^{3}$ Department of Bioproducts and Biosystems, Aalto University, Espoo, Finland
}

The importance of using biomimicking membranes for various biological applications is rising, as such models are relevant for imitating real organisms. In addition, biomimicking membranes are usually much more repeatable in preparation and easier to handle during analysis than real organisms or biological membranes. In this work, we developed a method for the adsorption of intact small unilamellar Escherichia coli (E. coli) vesicles (Z-average size of $73 \mathrm{~nm}$ ) on $\mathrm{SiO}_{2}$ substrate material. We describe the adsorption process based on the use of two surface sensitive techniques, i.e., nanoplasmonic sensing (NPS) and quartz crystal microbalance (QCM). The acquired data show that the adsorption follows a two-step process. The first step is a slow adsorption of $E$. coli vesicle aggregates held together by $5 \mathrm{mM}$ of calcium (Z-average size of $531 \mathrm{~nm}$ ). The Z-average of the aggregates decreased almost three times when the calcium concentration was decreased to $0.1 \mathrm{mM}$. This suggests that the aggregates were disassembling to some extent when calcium was removed from the system. With both techniques, i.e., NPS and QCM, we observed a second rapid adsorption step after the solution was changed to deionized water. In this second step, the aggregates started to fall apart as the calcium concentration dropped, and the released vesicles started to adsorb onto unoccupied spots at the $\mathrm{SiO}_{2}$ surface of the sensors. Extensive release of mass from the surface was confirmed by QCM, where it was reflected by a sharp increase of frequency, while NPS, due to its lower sensing depth of a few tens of nanometers, did not record such a change. Taken together, we have developed a protocol to form a supported vesicle layer (SVL) of $E$. coli vesicles on $\mathrm{SiO}_{2}$ surface using sodium 4-(2-hydroxyethyl)piperazine-1ethanesulfonate buffer, thus enabling the preparation of E. coli biomimicking SVLS for interaction studies of compounds of interest. The immobilization happens via a two-step adsorption process.

Keywords: aggregation, biomembrane, Escherichia coli, nanoplasmonic sensing, vesicles, quartz crystal microbalance 


\section{INTRODUCTION}

Reconstruction of membranes from lipids, which are extracted from real organisms, provides a useful tool for studying interactions of biomimicking membranes and xenobiotics of high biological relevance. To investigate such interactions, new methodologies are required to immobilize membranes on the surface of a particular sensor material. We present a new methodology, in which we utilize lipid vesicles composed of Escherichia coli (E. coli) total lipid extract as the biomimicking agent. E. coli vesicles immobilize on the utilized $\mathrm{SiO}_{2}$ sensor surface as a supported vesicle layer (SVL). The immobilization happens via a reversible aggregation mechanism, controlled by the concentration of calcium ion.

Recently, we developed a method for the immobilization of egg phosphatidylcholine (PC) onto $\mathrm{SiO}_{2}$ nanoplasmonic sensing (NPS) sensor surfaces as supported lipid bilayers (SLBs) and SVLs (Duša et al., 2018). However, the phosphatidyl choline mixture from chicken egg is substantially different from the rather complex E. coli total lipid extract. According to the manufacturer, $24.9 \%$ of the total mass of E. coli extract is composed of negatively charged phosphatidylglycerol and doubly negatively charged cardiolipin. Since the net surface charge of a $\mathrm{SiO}_{2}$ coated sensor is negative at $\mathrm{pH} 7.4$, the adsorption of highly negatively charged E. coli vesicles to $\mathrm{SiO}_{2}$ surface is prevented by electrostatic repulsion. It has previously been shown that cardiolipin is an important lipid in the membrane composition of E. coli lipid extracts (Lopes et al., 2010) and its content has a major effect on the membrane structure and dynamics (Unsay et al., 2013). Macdonald and Seelig showed that there is an excess of calcium ions on a surface of biomembranes composed of mixed cardiolipin and PC (Macdonald and Seelig, 1987). Even a very low concentration of calcium $(<2 \mathrm{mM})$ is able to shift the membrane surface potential from highly negative to positive values. Moreover, it could be well-anticipated that the total extract of lipids from E. coli incorporates lipopolysaccharides (LPS), which form the outer leaflet of the outer membrane in vivo (Lind et al., 2015). Therefore, LPS are also very important in mimicking the E. coli biomembrane system (Clifton et al., 2015; Lind et al., 2015). Clifton et al. found that divalent cations, such as calcium and magnesium, are able to form a stable cross-link with negatively charged LPS (Clifton et al., 2015). Lind et al. recently presented a method for the preparation of E. coli total lipid extract $\mathrm{SLB}$ on a $\mathrm{SiO}_{2}$ surface using TRIS buffer containing $\mathrm{CaCl}_{2}$ (Lind et al., 2015). However, to the best of our knowledge, there is no published method describing the formation of SVL from E. coli total lipid extracts on a $\mathrm{SiO}_{2}$ surface. The aim of this study is to describe the immobilization mechanism of E. coli vesicles modulated by the presence of calcium in 4-(2hydroxyethyl)piperazine-1-ethanesulfonic acid (HEPES) buffer, using data from the surface sensing techniques, NPS and quartz crystal microbalance (QCM).

\section{MATERIALS AND METHODS}

\section{Chemicals}

E. coli total lipid extract (PN 100500) in chloroform was purchased from Avanti Polar Lipids (Alabaster, AL, USA). Nitric acid (70\%) was obtained from Sigma (Darmstadt, Germany). Calcium chloride, ethylene glycol, HEPES, and HPLC-grade methanol were from VWR International Oy (Espoo, Finland). The $\mathrm{pH}$ calibration solutions (7.0 and 10.0) were obtained from Merck (Darmstadt, Germany). Distilled water was further purified with a Millipore water purification system (Millipore, Molsheim, France).

\section{Buffer Preparation}

HEPES acid concentration along with the concentration of $\mathrm{NaOH}$ were calculated with the Peakmaster 5.3 program (available for free at http://web.natur.cuni.cz/gas/) to reach a $\mathrm{pH}$ of 7.4 and an ionic strength of $10 \mathrm{mM}$. The resulting solution prepared from the calculated masses of HEPES and $\mathrm{NaOH}$ was checked by a $\mathrm{pH}$ meter to confirm that the $\mathrm{pH}$ of the buffer was within the range of $\mathrm{pH} 7.4 \pm 0.05$. A stock solution of $500 \mathrm{mM}$ of $\mathrm{CaCl}_{2}$ was used for dilution into sodium HEPES buffer with or without liposomes to obtain a final concentration of $5 \mathrm{mM}$ of $\mathrm{CaCl}_{2}$ in the buffer. All solutions were filtered through a polytetrafluoroethylene syringe filter with a $0.45 \mu \mathrm{m}$ pore size (Gelman Sciences, Ann Arbor, MI, USA).

\section{Vesicle Preparation}

The vesicles were prepared in the same manner as described in our previous study (Duša et al., 2018). Briefly, the calculated amount of $32.46 \mathrm{mM}$ stock solution of $E$. coli total lipid extract dissolved in chloroform was transferred to a glass vial. A thin film of lipids was produced by evaporating chloroform under a gentle stream of air. Traces of chloroform were further removed by overnight evacuation in a desiccator. Subsequently, lipids were rehydrated in HEPES buffer at $60^{\circ} \mathrm{C}$ for $60 \mathrm{~min}$ and continuously shaken to produce an E. coli multilamellar vesicle (MLV) dispersion with $4 \mathrm{mM}$ lipid concentration. Small unilamellar vesicles (SUV) were prepared from the MLV dispersion by 20 min sonication at $37 \mathrm{kHz}$ and $100 \%$ power in an ultrasonic bath ELMASONIC P $30 \mathrm{H}$ (Elma, Singen, Germany). After sonication, the lipid dispersion was filtered through a syringe filter of $0.45 \mu \mathrm{m}$ pore size to remove any dust particles. The size distribution of the SUVs was routinely checked by dynamic light scattering (DLS) with a Zetasizer Nano ZS instrument (Malvern Instruments, Malvern, UK).

\section{Nanoplasmonic Sensing Measurements}

NPS measurements based on localized surface plasmon resonance were conducted in optical transmission mode on $\mathrm{SiO}_{2}$ coated $(\sim 10 \mathrm{~nm})$ golden nanodisks supported on glass sensor chips. An Insplorion XNano II instrument (Insplorion AB, Gothenburg, Sweden) (Jackman et al., 2014) with a $1 \mathrm{~Hz}$ signal recording frequency was used for all NPS measurements. New sensors were cleaned by UV ozonization (UVC-1014 NanoBioAnalytics, Berlin, Germany) prior to their first use. The bulk refractive index (RI) sensitivity of a new sensor was checked by increasing concentrations of ethylene glycol diluted in water. The sensor cleaning process after every analysis consisted of gentle sonication in methanol at $80 \mathrm{kHz}$ and $30 \%$ power in sweep mode for $5 \mathrm{~min}$. All experiments were performed under continuous flow of $100 \mu \mathrm{L} / \mathrm{min}$, controlled by a REGLO Digital peristaltic pump (Ismatec, Wertheim, Germany) 
at $25^{\circ} \mathrm{C}$ (thermostated temperature). Data was collected with the Insplorer version 1.2 software (Insplorion $\mathrm{AB}$, Gothenburg, Sweden). OriginPro 8.6 software (OriginLab, Northampton, MA, USA) was used for evaluation of the acquired data.

\section{Quartz Crystal Microbalance Measurements}

A QCM unit with a polytetrafluorethylene flow cell (KEVA, Brno, Czech Republic) was used for analysis. $10 \mathrm{MHz}$ quartz crystals (International crystal manufacturing, Oklahoma City, OK, USA) with golden electrodes coated by $50 \mathrm{~nm}$ of $\mathrm{SiO}_{2}$ were used and the signal collection rate was $10 \mathrm{~Hz}$. The cell was thermostated to $25^{\circ} \mathrm{C}$ and a REGLO digital peristaltic pump, interfaced with an RS232 port to a PC, was used for maintaining the flow rate at $100 \mu \mathrm{L} / \mathrm{min}$. At the inlet side the peristaltic pump tubing was connected to a 10 -port selector valve (VICI AG International, Schenkon, Switzerland), which was operated by a Cole-Parmer Data Acquisition Module 18200-00 (ColeParmer, Vernon Hills, IL, USA). The fluidic setup and QCM data acquisition was further controlled by a custom designed Labview program (National Instruments, Austin, TX, USA). OriginPro 8.6 software (OriginLab, Northampton, MA, USA) was used for evaluation of the acquired data.

\section{RESULTS AND DISCUSSION \\ Vesicle Size and Aggregation}

After multiple sonication steps of MLV dispersions of $E$. coli extract vesicles, we observed a change of the dispersion from opalescent to clear. This suggests formation of SUVs. However, determination of the vesicle size showed that there were two different coexisting size populations. The size volume distribution demonstrated that $\sim 90 \%$ of the vesicles had an average size of $35 \mathrm{~nm}$ with a Z-average of $73.1 \pm 1.7 \mathrm{~nm}$.

In our recent study, eggPC vesicles diluted in sodium HEPES buffer (Na-HEPES) formed a SVL on a $\mathrm{SiO}_{2}$ sensor surface after treatment of the surface with $2 \mathrm{M} \mathrm{HNO}_{3}$. In contrast, eggPC SLB was formed on the same surface using Na-HEPES buffer containing $5 \mathrm{mM} \mathrm{CaCl}_{2}$ (Ca-HEPES) (Duša et al., 2018). In the case of $E$. coli vesicles no SVL was formed when using the same $\mathrm{HNO}_{3}$ treatment with Na-HEPES (data not shown). However, when Ca-HEPES was used to dilute the E. coli vesicle dispersion, the original solution turned from clear to turbid. This unexpected change indicated aggregation of E. coli vesicles. When this turbid dispersion was analyzed by DLS, one well-defined peak with a Z-average of $531.6 \pm 12.0 \mathrm{~nm}$ was observed, proving aggregation of $E$. coli vesicles. In addition, the cloudiness of the solution after adding Ca-HEPES did not recede after sonication at $37 \mathrm{kHz}$ for $10 \mathrm{~min}$ at $100 \%$ power setting.

The fast aggregation of $E$. coli vesicles was most probably caused by a high mass fraction $9.8 \%$ of cardiolipin, which possess two negatively charged phosphate groups. Calcium as a fusogenic agent is strongly linked to negatively charged phospholipids, promoting aggregation (Reviakine et al., 2000; Binder and Zschörnig, 2002; Agafonov et al., 2003).

It has been shown that $9 \mathrm{mM}$ calcium was able to fuse vesicles made of an equimolar ratio of cardiolipin and
eggPC. The rate of fusion increased with increasing calcium concentration (Wilschut et al., 1982). Moreover, polymorphisms were found to be induced in a biomembrane composed of cardiolipin/eggPC upon interaction with calcium (Unsay et al., 2013). Furthermore, Lemmin et al. performed computer simulations and demonstrated that cations were able to neutralize most of the negative charges of cardiolipin-rich biomembranes (Lemmin et al., 2013). Fusion of vesicles is generally an irreversible process when vesicles of a larger hydrodynamic diameter are produced (Ohki and Arnold, 2000). However, we were able to see a certain level of reversibility, when the calcium concentration was decreased in the solution. The dilution of $5 \mathrm{mM}$ calcium in HEPES with $0.15 \mathrm{mM}$ of $E$. coli vesicles was done with either water or HEPES buffer. The final concentration of calcium was $0.1 \mathrm{mM}$ and the concentration of vesicles was $3 \mu \mathrm{M}$ (50 times dilution). With both diluting solutions the Z-average dropped to $182.8 \mathrm{~nm}$ and $156.4 \mathrm{~nm}$ using water or HEPES buffer, respectively. This finding suggests that even though fusion of vesicles might be present during the aggregation process, the major part of the aggregation mechanism is reversible. Therefore, we speculate that SUVs form aggregates held together by bidental calcium cations. However, the interaction can be disrupted by decreasing the concentration of calcium when the aggregates disassemble back into SUVs. Considering a maximum packing sphere density occupying $74 \%$ of the available volume, one spherical $500 \mathrm{~nm}$ aggregate could encompass more than two thousands $35 \mathrm{~nm}$ E. coli vesicles. The structure and formation kinetics of vesicle aggregates are complicated, and therefore sophisticated methods and models have to be employed to reveal the actual structure (Roldán-Vargas et al., 2009).

The presence of LPS in the E. coli total extract might also play a role in the aggregation process as $17.6 \%$ of the total lipid mass was not identified by the producer. A previous study has shown that the LPS outer leaflet of the outer membrane lost its ordered structure when the divalent cations $(5 \mathrm{mM}$ calcium) were removed by the addition of $3 \mathrm{mM}$ EDTA and substituted with sodium cations (Clifton et al., 2015). The content of LPS in E. coli total extract membrane of SUVs was probably too low to form a compact leaflet and, as a result, the LPS molecules were scarcely distributed in both leaflets of the vesicle membrane. However, the addition of calcium could induce a reverse mechanism to the one described in Clifton et al. (2015). Flip-flop of LPS molecules to the outer leaflet and migration in membrane would form LPS-rich regions on the outer leaflet of the SUV membrane. This could in turn enable connection between LPS of two or more different SUVs, forming aggregates. It should be noted that the disaggregation observed in our study started after simple dilution of calcium concentration compared to the addition of EDTA to the system shown in Clifton et al. (2015).

The applied buffer in the immobilization might also play a major role in the aggregation process. Aggregation of E. coli vesicles was not observed when $2 \mathrm{mM} \mathrm{MgCl}_{2}$ was introduced to the vesicle dispersion (White et al., 2000), as the authors used a phosphate buffer system in contrast to Na-HEPES buffer used for dilution in this study. In addition, the original vesicle dispersion 
contained much bigger vesicles with hydrodynamic diameters larger than $160 \mathrm{~nm}$.

\section{NPS Analysis of Aggregates}

To confirm the reversibility of aggregation, the dynamic interaction of calcium with vesicles was further analyzed by NPS. At the beginning, the NPS signal of $E$. coli vesicle immobilization showed no adsorption onto the $\mathrm{SiO}_{2}$ sensor (Figure 1). However, a slow drift amounting $10.9 \pm 1.7 \cdot 10^{-3} \mathrm{~nm} / \mathrm{min}$ was observed at longer immobilization time. The total peak shift change after $65 \mathrm{~min}$ of solution introduction was $0.78 \mathrm{~nm}$ (Figure 1, red trace). This implies a very sparse layer of supported vesicles and also a very slow adsorption rate of $E$. coli vesicles using CaHEPES. Rinsing E. coli aggregates with Na-HEPES produced a step in the signal, but no other change was observed with continual rinsing. Surprisingly, upon the last rinsing step with MilliQ water, the initial drop in the signal was in course of $20 \mathrm{~min}$ substituted by a sharp increase in the peak shift (amounting 1.01 $\mathrm{nm} / \mathrm{min}$ at its maximum rate). The rise of peak shift started to get slower exponentially after the initial linear increase. This suggests that the initial high rate of adsorption on the unoccupied surface of the NPS sensor got gradually slower due to sterical hindrance as the surface got more densely covered with vesicles. It is very important to note that no vesicles were introduced to the system with the water rinse step. This is also clear from the maximum extinction in Figure 1 (gray and light pink), which increased due to scattering of light by aggregates. However, after a change of the rinsed solution to Na-HEPES or water the maximum extinction returned to the starting level. Therefore, the increase of the NPS signal at this water rinsing step had to be due to the disassembly of the immobilized aggregates, which released E. coli vesicles with dilution of calcium. This finding is further supported by the already presented analysis of the hydrodynamic diameters of the vesicles.

The kinetics of adsorption observed from the change in the NPS signals suggest that the aggregates most probably started to disassemble as calcium cations were rinsed off the surface. As the aggregates fell apart, the concentration of free vesicles near the $\mathrm{SiO}_{2}$ surface sharply increased. They started to occupy the free space between the aggregates, which was observed as a steep change in the peak shift. It is probable that the negative biomembrane surface charge was still neutralized by calcium cations. Due to the sensing depth of NPS of only a few tens of nanometers (Jackman et al., 2017a), disassembly was not observed by this technique. The short sensing depth is also the reason why a considerable change in the peak shift was not observed during adsorption of the vesicle aggregates.

A recent study has shown, that the concentration of calcium has a very strong effect on the formation of SLB from E. coli vesicles immobilized on a mercaptopropionic acid modified gold surface (Márquez and Vélez, 2017). However, in that work the authors prepared vesicles from E. coli polar lipid extracts. The lipid composition in this case was free from non-polar lipids and especially LPS would be removed during the extraction process. The authors observed that E. coli vesicles formed a full SLB, when the calcium concentration was increased from zero to $2 \mathrm{mM}$. When the concentration of calcium was further increased to $5 \mathrm{mM}$, only a partial SLB was formed. The SLB formation using $5 \mathrm{mM}$ of calcium was very similar to that observed using $0.5 \mathrm{mM}$ calcium, where also only a partial SLB was formed. It was speculated that the effect observed upon adding $5 \mathrm{mM}$ of calcium was the sum of SLB formation and adsorption of intact vesicles on the formed SLB. This speculation was confirmed by atomic force microscopy analysis. The analysis showed that $E$. coli extracted lipids formed a less even SLB, compared to that formed by eggPC and phosphatidic acid in a molar ratio of 90:10 (Márquez and Vélez, 2017). The formation of SLB was not observed in our experiments, which can be confirmed by the absence of the characteristic acceleration of NPS peak shift increase during the latter part of the immobilization (Jackman et al., 2016). This can be due to the different surface chemistry of the sensors used in these studies. Generally, the most often used surfaces in NPS applications include gold, $\mathrm{SiO}_{2}$, and $\mathrm{TiO}_{2}$ surfaces. The interaction of the lipid membranes with the mentioned surfaces follows different adsorption pathways depending whether gold, $\mathrm{SiO}_{2}$, or $\mathrm{TiO}_{2}$ coating is used ( $\mathrm{Zan}$ et al., 2014). However, even usage of the same surface with different buffers can alter the adsorption pathway. This was shown in our previous work on the use of phospholipid coatings in open tubular capillary electrochromatography (Hautala et al., 2003). Moreover, the difference in the composition between the liposomes prepared from total or polar lipid extracts discussed above might be another reason why the SLB formation was not observed.

Similarly to the analyzed vesicle system in our study, it has already been shown that polyion-induced aggregation of cationic vesicles preserve the integrity of vesicles without fusion of the vesicles (Bordi et al., 2006). Such clusters have the hydrodynamic radius defined by the equilibrium of short range attraction forces and long-range repulsion forces of polyelectrolyte-lipid clusters (Bordi et al., 2005). The largest aggregates were found at a 1:1 ratio of polyanions vs. cationic lipids (neutralization point) (Bordi et al., 2006). As the system presented in this study most probably includes a polyanionic core of LPS, the radius would be defined by the amount of calcium neutralization of the lipids and the LPS negative charge of $E$. coli vesicles. This also agrees with the finding that a decrease of calcium concentration increased the repulsion forces. As a result, the aggregates got smaller as shown by DLS analysis.

In a recent study conducted by Davydov et al. the aggregation vesicles composed of $\mathrm{PC} /$ cardiolipin and $\mathrm{PC} /$ cardiolipin/Brij 58 were investigated (Davydov et al., 2010). Different concentrations of the cationic polymer (polycation poly-Nethyl-4-vinylpyridinium bromide) were used to decrease the net charge of the vesicles, while the size of emerging aggregates was evaluated. The results demonstrated that when the net charge approached zero, the diameter of the aggregates reached a maximum value. The addition of Brij 58 to the vesicle prevented formation of any aggregates.

Cadmium cation, which is approximately of the same size as calcium cation, but has a higher electronegativity, was able to rigidify a liposomal membrane composed of negatively charged lipids with head groups composed of either phosphatidic acid, diphosphatidylglycerol (cardiolipin), phosphatidylglycerol, or phosphatidylserine (Kerek and 


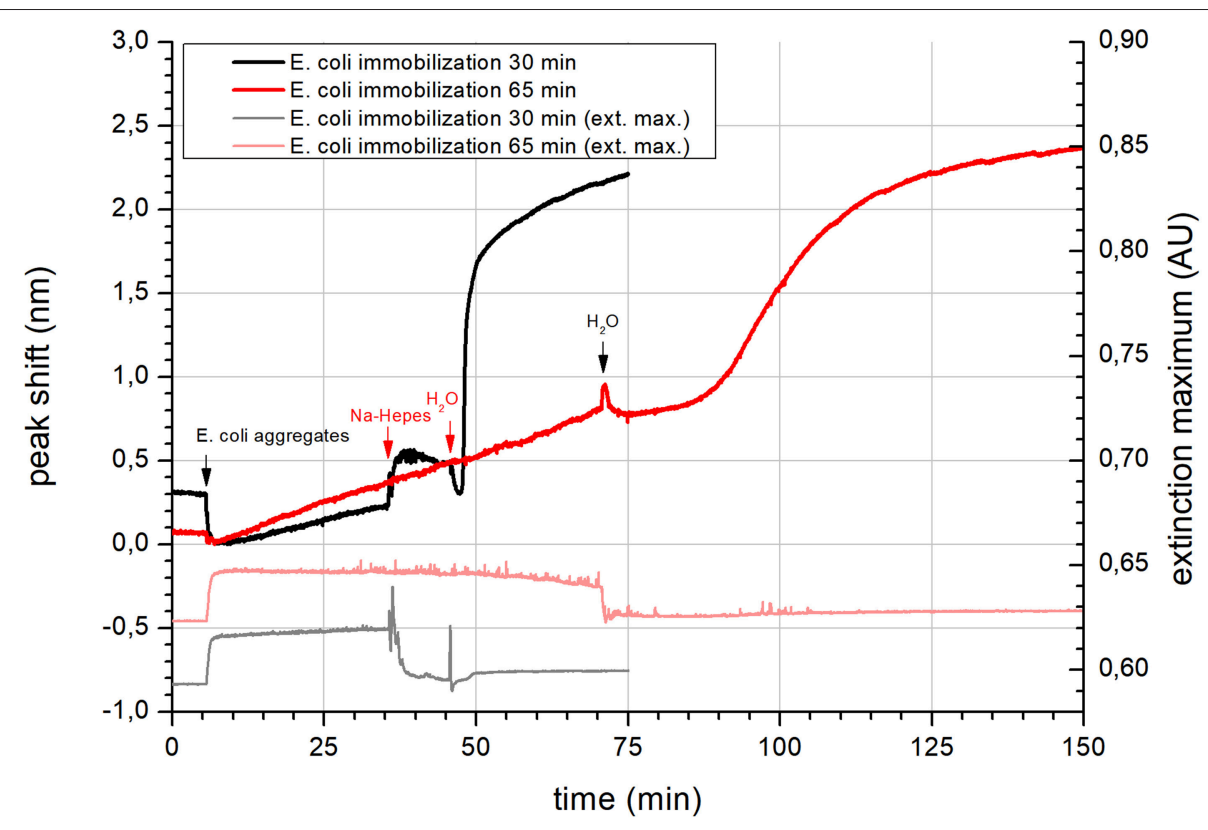

FIGURE 1 | NPS of the immobilization of E. coli lipid vesicles on a $\mathrm{SiO}_{2}$ coated sensor. Immobilization was conducted using E. coli lipid aggregates in Ca-HEPES (pH 7.4, Na-HEPES containing $5 \mathrm{mM}$ of $\mathrm{CaCl}_{2}$ ) at two different immobilization times (black trace-30 min and red trace-65 min). The left axis shows the peak shift data. Gray and light red traces are extinction maximum data respective to 30 and 65 min immobilization with the scale on the right axis. Application of different solutions is highlighted by black and red arrows with labels.

Prenner, 2016). Aggregates with diameters larger than $100 \mathrm{~nm}$ were observed only with phosphatidylserine, which was detected at the highest analyzed cadmium concentration of 2.0 mM. The kinetics of formation and structure of calciumphosphatidylserine aggregates was investigated by DLS. It was found that at a concentration region of $5-7 \mathrm{mM}$, the aggregation followed a limiting regime of rapid diffusion-limited aggregation (Roldán-Vargas et al., 2007).

Calcium is known to induce higher vesicle substrate interactions (Dacic et al., 2016) compared to the other divalent alkaline earth cations, like $\mathrm{Mg}^{2+}, \mathrm{Sr}^{2+}$, and $\mathrm{Ba}^{2+}$ and, therefore, one could expect that the aggregation effect would be less pronounced with other cations. The observed aggregation could also be a type of membrane appression, as discussed in a recent study (Jäger and Büchel, 2018) where the role of magnesium in the membrane stacking of thylakoids was studied. The authors mention the interplay of van der Waal's attraction forces, electrostatic interaction forces, and hydrostructural forces, which in sum determine the appression. Based on this finding, it is not straightforward to predict if changing the cation or increasing or decreasing the cation concentration would influence the resulting interaction. In this work, we therefore selected only one cation and one concentration to describe the observed aggregation phenomenon, before investigating other factors influencing the aggregation itself.

\section{QCM Analysis of Aggregates}

QCM was utilized to deeper investigate the adsorption process of $E$. coli vesicles. This surface sensitive technique detects the total mass of matter adsorbed on the sensor surface (Konradi et al., 2012) while NPS is able to detect adsorbed matter with a different RI than the background solution. Therefore, vesicles including the mass of the entrapped solution are detected with QCM, which is often used for evaluation of the type of formed lipid layer, i.e., SLB or SVL (Jackman et al., 2017b). Additionally, QCM has roughly one order of magnitude higher sensing depth compared to NPS, which extends a few hundred nanometers above the sensor surface. Scanning electron microscopy as well as atomic force microscopy would be very suitable techniques to decipher the morphology of the adsorbed aggregates, however, they do not provide continual analysis data for a dynamically changing systems. Only "snapshots" of disaggregation at particular decreasing concentrations of calcium would be obtained with such techniques. Based on the discussion above we decided that QCM would be the most suitable methodology to complement the NPS measurements.

When the vesicle aggregates reached the sizes of $500 \mathrm{~nm}$ it could be well-expected that the signal change observed by QCM would be more pronounced when aggregates were adsorbed. Even if the sensing depth of the $10 \mathrm{MHz}$ sensor reaches slightly below $200 \mathrm{~nm}$ (Rodahl and Kasemo, 1996), more than half of the aggregate is outside the sensing range. This presumption was well-followed during the experiment (Figure 2). The adsorption of the vesicle aggregates had again a linear character and a change in the adsorption rate was not observed even if the time for adsorption was prolonged from 40 to $160 \mathrm{~min}$ (Figure 2). The maximum recorded change in the frequency caused by $160 \mathrm{~min}$ aggregate adsorption was $1,007 \mathrm{~Hz}$. The second step of the adsorption started as soon as Na-HEPES 


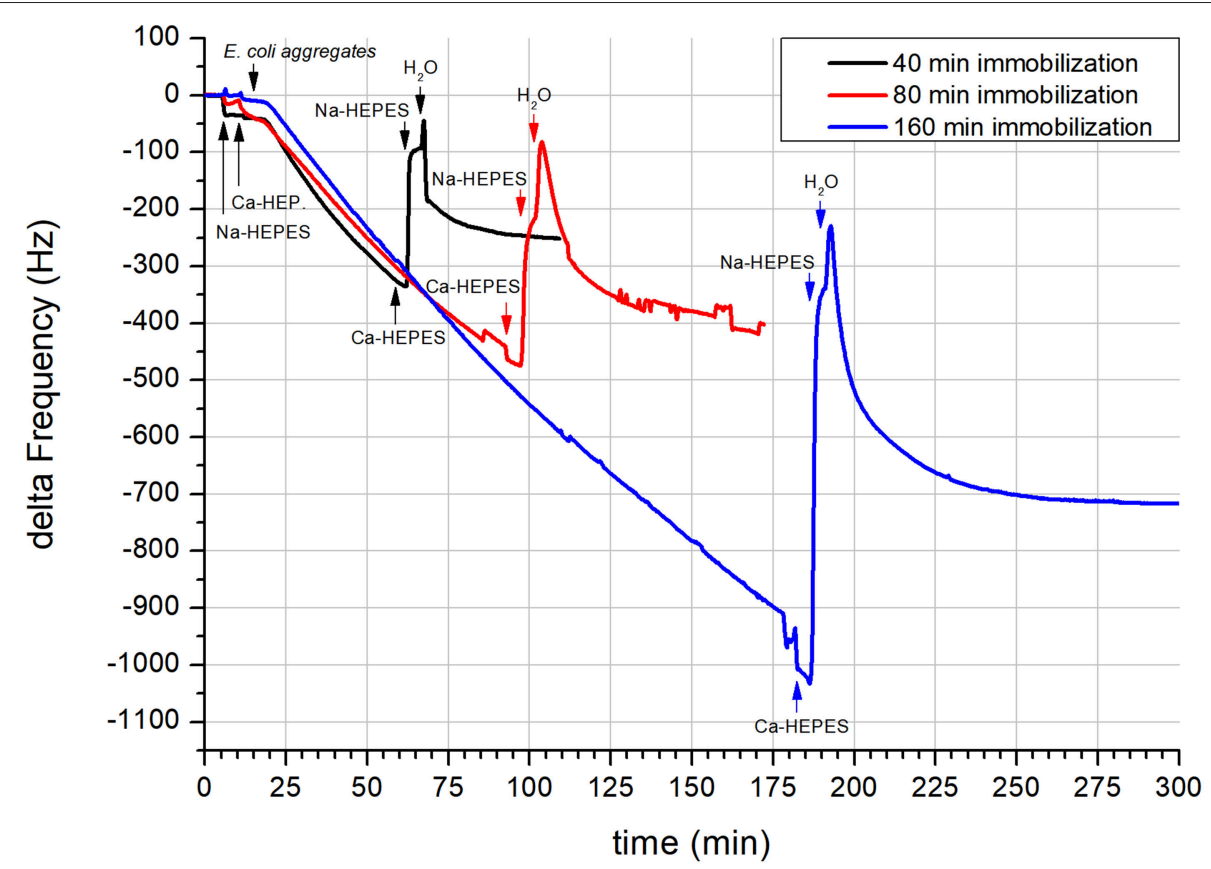

FIGURE 2 | QCM of the immobilization of E. coli lipid vesicles on a $10 \mathrm{MHz} \mathrm{SiO}_{2}$ coated sensor. Immobilization was conducted using E. coli lipid aggregates in Ca-HEPES ( $\mathrm{pH} 7.4$, Na-HEPES containing $5 \mathrm{mM}$ of $\mathrm{CaCl}_{2}$ ) at three different immobilization times (black trace-40 min, red trace-80 min, and blue trace-160 min). Application of different solutions is highlighted by black, red, and blue arrows with labels. All traces were smoothed during processing by OriginPro software using a Savitzky-Golay method (500 points) to remove subtle interferences from the electric power grid.

was introduced to the measurement cell, evidence by a rapid frequency increase of $801 \mathrm{~Hz}$ (Figure 2). This suggests that a considerable amount of the adsorbed mass was removed from the sensor surface. However, upon a subsequent water rinse, the frequency started to rapidly decrease. The observed exponential decrease of the frequency stabilized in over $100 \mathrm{~min}$ at a value of $-716 \mathrm{~Hz}$. The higher frequency $(-716 \mathrm{~Hz})$ of the resulting adsorbed system compared to adsorbed aggregates $(-1017 \mathrm{~Hz})$ suggests that the thickness of the final adsorbed layer was considerably smaller than the layer of adsorbed aggregates. It should also be noted that the aggregate adsorption stopped before reaching full saturation of the sensor surface. Therefore, the frequency of the surface saturated with aggregates would be still lower. Due to the higher risk of bubble formation during very long QCM runs, we did not continue with longer immobilization times.

In Figure 2, the subsequent experiments using prolonged times of adsorption also show the dependency of the final frequency of the vesicle layer to the aggregate immobilization time (see Table 1). From the table it is clear that the dependency is not linear and decreases with longer immobilization times. The reason for that might be increased saturation of the sensor surface with higher number of released SUV. Due to the more pronounced competition of SUV for adsorption, a bigger part of the released SUV is rinsed from the measurement cell. This in turn results in a lower factor of final frequency change per minute of aggregate immobilization.

The QCM results are in good agreement with the NPS measurements. Both methods exhibited similar shape of the
TABLE 1 | Dependency of final frequency change of the developed supported vesicle layer on the time of aggregate immobilization.

\begin{tabular}{ccc}
\hline $\begin{array}{c}\text { Aggregate } \\
\text { immobilization time } \\
\text { (min) }\end{array}$ & $\begin{array}{c}\text { Total frequency } \\
\text { change }(\mathbf{H z})\end{array}$ & $\begin{array}{c}\text { Factor of frequency change } \\
\text { per minute of aggregate } \\
\text { immobilization }\end{array}$ \\
\hline 40 & 250 & 6.25 \\
80 & 408 & 5.10 \\
160 & 716 & 4.48 \\
\hline
\end{tabular}

adsorption curve (however in mirrored direction). The QCM data therefore complement to high extent the observed NPS data.

\section{CONCLUSIONS}

Both analysis systems (NPS and QCM) together with DLS analysis enable to describe the adsorption mechanics of formation of $E$. coli SVL from vesicle aggregates. The process, based on the observed results and literature data, is most probably governed by a variation of the calcium concentration.

Our results confirm that a considerable part of the lipid aggregates disassembled or was released from the surface. Concurrent adsorption of the released vesicles quickly occupied the free space on the sensor surface when water was applied. The reaction of the system to rinsing with water was immediate. However, because most of the vesicles would be carried away from the surface by a constant flow, the disassembly of a portion 
of the aggregates had to be of quite slow dynamics in order to be able to supply free vesicles over the $100 \mathrm{~min}$ range of the observed SVL adsorption. On the basis of the acquired data we suggest that while the rinsing continued, the aggregates were totally deconstructed and only a SVL was left.

To conclude, in this study we have shown that E. coli vesicles can be immobilized on $\mathrm{SiO}_{2}$ sensors using $\mathrm{Ca}$ HEPES buffer. The immobilization followed a two-step adsorption process. Successful immobilization of E. coli vesicles on the $\mathrm{SiO}_{2}$ sensor surface enables future analysis of interactions between $E$. coli biomimicking SVL and target compounds.

\section{DATA AVAILABILITY}

The raw data supporting the conclusions of this manuscript will be made available by the authors, without undue reservation, to any qualified researcher.

\section{REFERENCES}

Agafonov, A., Gritsenko, E., Belosludtsev, K., Kovalev, A., Gateau-Roesch, O., Saris, N.-E., et al. (2003). A permeability transition in liposomes induced by the formation of Ca2+/palmitic acid complexes. Biochim. Biophys. 1609, 153-160. doi: 10.1016/S0005-2736(02)00666-1

Binder, H., and Zschörnig, O. (2002). The effect of metal cations on the phase behavior and hydration characteristics of phospholipid membranes. Chem. Phys. Lipids 115, 39-61. doi: 10.1016/S0009-3084(02)00005-1

Bordi, F., Cametti, C., Diociaiuti, M., and Sennato, S. (2005). Large equilibrium clusters in low-density aqueous suspensions of polyelectrolyteliposome complexes: a phenomenological model. Phys. Rev. E 71:050401. doi: 10.1103/PhysRevE.71.050401

Bordi, F., Cametti, C., Sennato, S., and Diociaiuti, M. (2006). Direct evidence of multicompartment aggregates in polyelectrolyte-charged liposome complexes. Biophys. J. 91, 1513-1520. doi: 10.1529/biophysj.106.085142

Clifton, L. A., Holt, S. A., Hughes, A. V., Daulton, E. L., Arunmanee, W., Heinrich, F., et al. (2015). An accurate in vitro model of the E. coli envelope. Angew. Chemie Int. Ed. 54, 11952-11955. doi: 10.1002/anie.201504287

Dacic, M., Jackman, J. A., Yorulmaz, S., Zhdanov, V. P., Kasemo, B., and Cho, N.-J. (2016). Influence of divalent cations on deformation and rupture of adsorbed lipid vesicles. Langmuir 32, 6486-6495. doi: 10.1021/acs.langmuir.6b00439

Davydov, D. A., Rakhnyanskaya, A. A., Orlov, V. N., Bychkova, A. V., Kovarskii, A. L., and Yaroslavov, A. A. (2010). Complexes of anionic liposomes and a cationic polymer: composition, structure, and characteristics. Polym. Sci. Ser. A 52, 693-703. doi: 10.1134/S0965545X10070035

Duša, F., Chen, W., Witos, J., and Wiedmer, S. K. (2018). Nanoplasmonic sensing and capillary electrophoresis for fast screening of interactions between phosphatidylcholine biomembranes and surfactants. Langmuir 34, 5889-5900. doi: 10.1021/acs.langmuir.8b01074

Hautala, J. T., Lindén, M. V., Wiedmer, S. K., Ryhänen, S. J., Säily, M. J., Kinnunen, P. K., et al. (2003). Simple coating of capillaries with anionic liposomes in capillary electrophoresis. J. Chromatogr. A 1004, 81-90. doi: 10.1016/S0021-9673(03)00570-3

Jackman, J. A., Rahim Ferhan, A., and Cho, N.-J. (2017a). Nanoplasmonic sensors for biointerfacial science. Chem. Soc. Rev. 46, 3615-3660. doi: 10.1039/C6CS00494F

Jackman, J. A., Špačková, B., Linardy, E., Kim, M. C., and Yoon, B. K., Homola, J., et al. (2016). Nanoplasmonic ruler to measure lipid vesicle deformation. Chem. Commun. 52, 76-79. doi: 10.1039/c5cc06861d

Jackman, J. A., Yorulmaz Avsar, S., Ferhan, A. R., Li, D., Park, J. H., Zhdanov, V. P., et al. (2017b). Quantitative profiling of nanoscale liposome deformation

\section{AUTHOR CONTRIBUTIONS}

FD, JW, WC, and SW proposed and designed the study on the basis of experimental NPS results obtained by WC. FD wrote the first draft and performed QCM and DLS experiments. SW supervised the study and critically revised the manuscript. All authors contributed to the process of manuscript revision, read, and approved the submitted version.

\section{FUNDING}

This work was supported by the Programme for research and mobility support of starting researchers of the Czech Academy of Sciences number MSM200311602 (FD) and by institutional support RVO:68081715 (FD). Financial support from the University of Helsinki, Faculty of Science, is acknowledged (SW).

by a localized surface plasmon resonance sensor. Anal. Chem. 89, 1102-1109. doi: 10.1021/acs.analchem.6b02532

Jackman, J. A., Zhdanov, V. P., and Cho, N. J. (2014). Nanoplasmonic biosensing for soft matter adsorption: kinetics of lipid vesicle attachment and shape deformation. Langmuir 30, 9494-9503. doi: 10.1021/la502431x

Jäger, S., and Büchel, C. (2018). Cation-dependent changes in the thylakoid membrane appression of the diatom Thalassiosira pseudonana. Biochim. Biophys. Acta 1860, 41-51. doi: 10.1016/j.bbabio.2018.11.003

Kerek, E. M., and Prenner, E. J. (2016). Inorganic cadmium affects the fluidity and size of phospholipid based liposomes. Biochim. Biophys. Acta 1858, 3169-3181. doi: 10.1016/j.bbamem.2016.10.005

Konradi, R., Textor, M., and Reimhult, E. (2012). Using complementary acoustic and optical techniques for quantitative monitoring of biomolecular adsorption at interfaces. Biosensors 2, 341-376. doi: 10.3390/bios2040341

Lemmin, T., Bovigny, C., Lançon, D., and Dal Peraro, M. (2013). Cardiolipin models for molecular simulations of bacterial and mitochondrial membranes. J. Chem. Theory Comput. 9, 670-678. doi: 10.1021/ct300590v

Lind, T. K., Wacklin, H., Schiller, J., Moulin, M., Haertlein, M., Pomorski, T. G., et al. (2015). Formation and characterization of supported lipid bilayers composed of hydrogenated and deuterated Escherichia coli lipids. PLoS ONE 10:e0144671. doi: 10.1371/journal.pone.0144671

Lopes, S., Neves, C. S., Eaton, P., and Gameiro, P. (2010). Cardiolipin, a key component to mimic the $E$. coli bacterial membrane in model systems revealed by dynamic light scattering and steady-state fluorescence anisotropy. Anal. Bioanal. Chem. 398, 1357-1366. doi: 10.1007/s00216-010-4028-6

Macdonald, P. M., and Seelig, J. (1987). Calcium binding to mixed cardiolipinphosphatidylcholine bilayers as studied by deuterium nuclear magnetic resonance. Biochemistry 26, 6292-6298. doi: 10.1021/bi00393a050

Márquez, I. F., and Vélez, M. (2017). Formation of supported lipid bilayers of charged E. coli lipids on modified gold by vesicle fusion. MethodsX 4, 461-468. doi: 10.1016/j.mex.2017.11.002

Ohki, S., and Arnold, K. (2000). A mechanism for ion-induced lipid vesicle fusion. Colloids Surf. B 18, 83-97. doi: 10.1016/S0927-7765(99)00131-9

Reviakine, I., Simon, A., and Brisson, A. (2000). Effect of Ca 2+ on the morphology of mixed DPPC-DOPS supported phospholipid bilayers. Langmuir 16, 1473-1477. doi: 10.1021/la990806g

Rodahl, M., and Kasemo, B. (1996). On the measurement of thin liquid overlayers with the quartz-crystal microbalance. Sens. Actuat. A 54, 448-456. doi: 10.1109/SENSOR.1995.721939

Roldán-Vargas, S., Barnadas-Rodríguez, R., Quesada-Pérez, M., Estelrich, J., and Callejas-Fernández, J. (2009). Surface fractals in liposome aggregation. Phys. Rev. E 79:011905. doi: 10.1103/PhysRevE.79.011905 
Roldán-Vargas, S., Martín-Molina, A., Quesada-Pérez, M., Barnadas-Rodríguez, R., Estelrich, J., and Callejas-Fernández, J. (2007). Aggregation of liposomes induced by calcium: a structural and kinetic study. Phys. Rev. E 75:021912. doi: 10.1103/PhysRevE.75.021912

Unsay, J. D., Cosentino, K., Subburaj, Y., and García-Sáez, A. J. (2013). Cardiolipin effects on membrane structure and dynamics. Langmuir 29, 15878-15887. doi: $10.1021 / \mathrm{la} 402669 \mathrm{z}$

White, G. F., Racher, K. I., Lipski, A., Hallett, F. R., and Wood, J. M. (2000). Physical properties of liposomes and proteoliposomes prepared from Escherichia coli polar lipids. Biochim. Biophys. Acta. 1468, 175-186. doi: 10.1016/S0005-2736(00)00255-8

Wilschut, J., Holsappel, M., and Jansen, R. (1982). Ca2 ${ }^{+}$-induced fusion of cardiolipin/phosphatidylcholine vesicles monitored by mixing of aqueous contents. Biochim. Biophys. Acta 690, 297-301. doi: 10.1016/0005-2736(82)90334-0
Zan, G. H., Jackman, J. A., Kim, S. O., and Cho, N. J. (2014). Controlling lipid membrane architecture for tunable nanoplasmonic biosensing. Small 10, 4828-4832. doi: 10.1002/smll.201400518

Conflict of Interest Statement: The authors declare that the research was conducted in the absence of any commercial or financial relationships that could be construed as a potential conflict of interest.

Copyright (C) 2019 Duša, Chen, Witos and Wiedmer. This is an open-access article distributed under the terms of the Creative Commons Attribution License (CC BY). The use, distribution or reproduction in other forums is permitted, provided the original author(s) and the copyright owner(s) are credited and that the original publication in this journal is cited, in accordance with accepted academic practice. No use, distribution or reproduction is permitted which does not comply with these terms. 